\title{
HFE-Related Hemochromatosis: The Haptoglobin 2-2 Type Has a Significant but Limited Influence on Phenotypic Expression of the Predominant p.C282Y Homozygous Genotype
}

\author{
Gérald Le Gac, ${ }^{1,2,3}$ Chandran Ka, ${ }^{1,2,3}$ Isabelle Gourlaouen, ${ }^{1,2}$ Laurence Bryckaert, ${ }^{1}$ \\ Anne-Yvonne Mercier, ${ }^{1}$ Brigitte Chanu, ${ }^{1}$ Virginie Scotet, ${ }^{1,2}$ and Claude Férec ${ }^{1,2,3,4}$ \\ ${ }^{1}$ Etablissement Français du Sang Bretagne, Brest 29200, France \\ ${ }^{2}$ Inserm, U613, Brest 29200, France \\ ${ }^{3}$ Laboratoire de Génétique Moléculaire et d'Histocompatibilité, Hôpital Morvan, Centre Hospitalier Universitaire de Brest, \\ Brest 29200, France \\ ${ }^{4}$ Faculté de Médecine de Brest et des Sciences de la Santé, Université de Bretagne Occidentale, Brest 29200, France \\ Correspondence should be addressed to Gérald Le Gac, gerald.legac@univ-brest.fr
}

Received 29 October 2008; Revised 4 May 2009; Accepted 7 October 2009

Recommended by Abdullah Kutlar

\begin{abstract}
Phenotypic expression of the common p.C282Y/p.C282Y HFE-related hemochromatosis genotype is heterogeneous and depends on a complex interplay of genetic and non-genetic factors. Haptoglobin has a crucial role in free hemoglobin iron recovery, and exists as three major types: Hp1-1, Hp2-1 and Hp2-2. Hp2-2 favors endocytosis of hemoglobin iron in monocytes/macrophages, resulting in partial iron retention and increased intracellular ferritin levels. This situation is generally not expected to severely affect iron homeostasis, but was found to correlate with elevated serum iron indices in healthy men. Whether the Hp2-2 genotype acts as a modifier in HFE-related hemochromatosis is unclear. In this study we investigated influence of Hp2-2 and of potential confounders on the iron indices of $351 \mathrm{p}$.C282Y homozygous patients. We conclude that there is a cause-and-effect relationship between the Hp2-2 genotype and increased iron indices in p.C282Y homozygous patients. The Hp2-2 effect is, however, limited and only apparent in males.
\end{abstract}

Copyright (C) 2009 Gérald Le Gac et al. This is an open access article distributed under the Creative Commons Attribution License, which permits unrestricted use, distribution, and reproduction in any medium, provided the original work is properly cited.

\section{Introduction}

Hemochromatosis (HC; synonymous with hereditary hemochromatosis) refers to several inherited disorders of iron metabolism characterized by progressive iron accumulation in major organs [1-4]. If not recognized and treated, this progressive iron loading can lead to tissue damage and severe clinical complications, including cirrhosis, hepatocellular carcinoma, and cardiomyopathy [5].

The historical and predominant form of hemochromatosis is an adult-onset autosomal recessive condition usually associated with the HFE p.C282Y/p.C282Y genotype. In Northern European populations, this genotype is carried by approximately 1 person in 200 [4]. However, its phenotypic expression is heterogeneous and clearly depends on a balance between accentuating and reducing factors. The influence of gender, age, and lifestyle components, such as alcohol abuse, has been well documented [2, 6, 7]. Involvement of modifier genes or epigenetic mechanisms is less obvious, but is supported by studies in $\mathrm{Hfe}$ knockout mice, as well as by the greater concordance between biological and clinical iron overload phenotypes within families than between families $[4,8,9]$.

Most iron in the body is directed to erythropoiesis and is present as heme in hemoglobin (Hb). Hemoglobin iron is normally recycled after destruction of senescent erythrocytes by macrophages. Under physiological conditions, however, up to $10 \%$ of $\mathrm{Hb}$ molecules escape into the plasma [10]. These free $\mathrm{Hb}$ molecules are bound by haptoglobin $(\mathrm{Hp})$, which delivers them to the hepatic parenchymal cells, Kupffer 
cells and other macrophages. In this way, haptoglobin prevents iron losses through the glomeruli and protects the kidney against oxidative injury [11].

Haptoglobin is synthesized as a single polypeptide chain, which is cleaved in the endoplasmic reticulum to an aminoterminal $\alpha$-chain and a carboxy-terminal $\beta$-chain [12]. The basic structure of $\mathrm{Hp}$ is a homodimer designated $\mathrm{Hp} 1-$ 1 in which the two $\mathrm{Hp}$ molecules are joined by a single disulfide bridge through their respective $\alpha$-chains. Humans differ from other species by having two common $H p$ alleles (denoted Hp1 and $H p 2$ ), which, due to partial gene duplication, code for either a short or a long $\alpha$-chain, and the $\beta$-chain. Since the elongated $\alpha$-chain contains two cysteine residues that form disulfide bridges with two other $\alpha$-chains, individuals homozygous for the $H p 2$ allele show a multimeric Hp type designated Hp2-2. Hp2-1 refers to the type (both $\mathrm{Hp}$ dimers and multimers) seen in individuals heterozygous for the two $\mathrm{Hp}$ alleles [13].

$\mathrm{Hp} 2-2$ has been reported to favor endocytosis of $\mathrm{Hb}$ Hp complexes by monocytes/macrophages and to produce partial iron retention $[13,14]$. This results in higher intracellular ferritin concentrations, whereas the impact on serum iron parameters was found to be moderate and to reach significance only in healthy males [14]. In agreement with this gender-related effect, $\mathrm{Hp} 2-2$ was associated with elevated serum iron and ferritin levels in p.C282Y homozygous male patients but not in p.C282Y homozygous female patients [15]. However, this finding has been challenged by two other studies $[16,17]$.

Tolosano et al. have recently reported that $H f e$ and $H p$ compound knockout mice accumulated significantly less hepatic iron than $\mathrm{Hfe}$-null mice, thus demonstrating that $\mathrm{Hp}$-mediated $\mathrm{Hb}$-iron recovery may contribute to iron loading in $\mathrm{HC}$ [18]. Hp2 does not designate a null allele. Rather, it encodes a protein that could favor free hemoglobin iron delocalization into macrophages. To gain insight into the role of $\mathrm{Hp}$ as a modifier gene in HFE-related hemochromatosis, we investigated the influence of the $\mathrm{Hp} 2-2$ genotype in a large number of p.C282Y homozygous patients. We hypothesized that it might be limited and masked by the predominant effect of gender and other important non-genetic modifiers (age and alcohol abuse).

\section{Materials}

2.1. Study Patients. Three-hundred and fifty-one patients were enrolled in the study. At the first visit of these patients to a healthcare center in Brittany (western part of France), a clinical questionnaire was completed by the specialized physician. Information contained in this questionnaire was previously described in detail [6]. It provided information regarding sociodemographic characteristics of patients, their age at diagnosis, the circumstances of HC diagnosis, the biochemical parameters, and the clinical signs associated at the time of onset.

Basic reasons for diagnosis were presence of the HFE p.C282Y/p.C282Y genotype and the observation of abnormal iron indices (elevated transferrin saturation with or without elevated serum ferritin concentration). It means that not all patients presented with clinical signs.

The selected patients did not carry additional mutations on HAMP and HJV, two previously reported modifiers of the p.C282Y homozygous phenotype $[19,20]$.

This study complied with French bioethics regulations. Informed consent of patients was obtained before blood samples were taken.

\subsection{Assessment of the Amount of Iron Removed by Phlebotomy} and of Daily Alcohol Consumption. All patients were followed up in the same healthcare center, under regular practices (the targeted value for serum ferritin was $100 \mu \mathrm{g} / \mathrm{L}$ ).

The amount of iron removed by phlebotomy was assessed from the volume of blood extracted during depletion therapy, assuming that $500 \mathrm{~mL}$ of blood contains $250 \mathrm{mg}$ of elemental iron. A total of 26 patients ( 17 males and 9 females) had not completed iron depletion therapy when the study began. This subgroup of patients was excluded from all statistical analyses of the iron removed by phlebotomy variable.

Daily alcohol consumption was assessed from a detailed item included in the clinical questionnaire, which measured the amount of beer, wine, and liquor consumed, assuming respective alcohol (ethanol) contents of $4 \%, 12 \%$, and $20 \%$. Alcohol abuse was defined as consumption of $\geq 60$ g per day [21].

2.3. Haptoglobin Genotyping. Haptoglobin polymorphism was determined using two complementary allele-specific PCRs, as fully described by Koch and collaborators [22]. Briefly, a first PCR exploits the size difference due to the two extra exons of the $H p 2$ allele. It leads to the amplification of a 1757-bp product from the Hp1 allele and of a 3481-bp product from the $\mathrm{Hp} 2$ allele. Because of the primer binding sites and their $5^{\prime}$ to $3^{\prime}$ orientations, a second PCR permits the amplification of a 349-bp product from the $\mathrm{Hp} 2$ allele but no amplification from the $\mathrm{Hp} 1$ allele. This makes identification of the Hp2-1 heterozygous genotype more reliable as, in the first round of PCR, amplification of the HP1-specific 1757bp product is more efficient and occasionally leads to a very weak detection of the Hp2-specific 3481-bp product.

2.4. Statistical Analyses. Statistical analyses were performed using Epi-Info (version 6.04; Centers for Disease Control and Prevention, Atlanta, Ga, USA) or SAS (version 9.1; SAS Institute, Cary, NC) software.

Analysis of variances (ANOVA) and Student's $t$-test, considering either the pooled (equal variance) or the Satterthwaite (unequal variance) method, were used to test for between-group differences in means. Logarithmic transformation were used for all statistical analyses to ensure that serum ferrtin and iron removed by phlebotomy were approximately normally distributed.

The following potential confounders were considered in multiple linear regression models with either serum ferritin concentration or amount of iron removed by phlebotomy as dependent variable: $H p$ genotypes, gender, age at diagnosis 
(three dummy variables defined by the quartiles with quartile 1 as reference), and alcohol consumption (abusers versus non abusers). $H p$ genotype variable was assessed by grouping the Hp1-1 and Hp2-1 genotypes, and by only considering the Hp2-2 genotype as potential predictor. This allowed us to increase the size of the category used as reference. It should be noted that the $H p$ genotype variable was initially coded as two dummy variables (Hp2-1 versus $\mathrm{Hp} 1-1$ and $\mathrm{Hp} 2-2$ versus $\mathrm{Hp} 1-1$ ), and that by contrast with $\mathrm{Hp} 2-2, \mathrm{Hp} 2-1$ did not show any association with higher serum ferritin or iron removed by phlebotomy.

As serum ferritin concentration and iron removed by phlebotomy were $\log _{10}$ transformed, the regression coefficient $\beta$ corresponded to a $10^{\beta}$ times change in mean $\mathrm{Y}$ (i.e., serum ferritin or iron removed) per unit increment in the explanatory variable. In a multiple linear regression model, the coefficients $\beta$ are ajusted for the other covariables."

\section{Results}

3.1. Hp Genotype Distribution. Hp genotypes among the 351 p.C282Y homozygous were distributed as follows: 46 for Hp1-1 (13.1\%), 179 for Hp2-1 (51.0\%), and 126 for Hp2$2(35.9 \%)$. These genotype frequencies were in accordance with previous reports in Northern European populations $[15,17,23]$.

\subsection{Iron Overload Status Regarding Hp Genotypes}

3.2.1. Univariate Analyses. Analysis of variance failed to reveal any significant difference between the various $H p$ groups of p.C282Y homozygous patients, in terms of age at diagnosis, transferrin saturation, log-transformed serum ferritin, and log-transformed iron removed by phlebotomy (Table 1(a)).

These results contrasted with the positive effects of male sex and alcohol abuse on all the studied iron parameters (Tables 1(b) and 1(c); Student's $t$-tests).

3.2.2. Multivariate Analyses. Multiple linear regression models were fitted to test influence of $H p$ genotypes ( $\mathrm{Hp} 2-2$ versus Hp1-1 and Hp2-1) on either log-transformed serum ferritin or log-transformed iron removed by phlebotomy, after adjustment for gender, age at diagnosis, and alcohol consumption (Table 2). For both the dependent variables assessed, higher values were not only seen in male patients $(P<.0001$ and $P<.0001$, resp. $)$, patients of greater age at diagnosis $(P<.0001$ and $P<.0001)$ and patients who drank at least $60 \mathrm{~g}$ of alcohol per day $(P=.0073$ and $P=.0096)$ but also in patients with the Hp2-2 genotype $(P=.0033$ and $P=.0024)$.

With $\log _{10}$ transformation of the dependant variable (i.e. serum ferritin), the regression coefficient of the "Hp group" variable was 0.117 . It means that average serum ferritin was $10^{0.117}$ or 1.31 times higher in patients with $\mathrm{Hp} 2-2$ genotype than in patients with Hp1-1 or Hp1-2 genotype, this after adjustment on other covariables. The model also indicated that mean serum ferritin concentration appeared 1.57 times higher in alcohol abusers that in non alcohol abusers.

We did not observed an interaction between gender and Hp genotype ( $P=.5959)$. However, because gender was confirmed as the major modifier in phenotypic expression of the p.C282Y homozygous genotype, and because the effect of the Hp2-2 genotype on serum iron parameters was previously restricted to the male sex $[14,15]$, analyses were performed in men and women, separately.

\subsection{Iron Overload Status Regarding Gender and Hp Geno-} types. As only two of 27 heavy alcohol drinkers were females, patients who drank less than $60 \mathrm{~g}$ of alcohol per day were first considered. In this category, analysis of variance revealed significant differences in means of log-transformed serum ferritin and log-transformed iron removed by phlebotomy between the various $H p$ groups of males, but not of females (data not shown). As expected, subsequent pairwise $t$-tests ascribed the observed differences to higher serum ferritin and higher iron removed by phlebotomy in male carriers of the Hp2-2 genotype (data not shown).

Linear regression analysis indicated that these observations remained unchanged after adjustment for age at diagnosis and daily alcohol consumption (Tables 3(a) and $3(b))$.

\section{Discussion}

Only a small fraction of hemoglobin iron passes through the haptoglobin recovery pathway. For this reason, it is generally believed that $\mathrm{Hp}$ polymorphism does not severely affect iron homeostasis, and does not consistently explain the large phenotypic variability seen in carriers of the common HFE p.C282Y/p.C282Y genotype. However, Vlierberghe et al. have reported a striking association between $\mathrm{Hp} 2-2$ and more pronounced iron overload phenotypes in p.C282Y/p.C282Y males [15]. In view of this finding, and also bearing in mind contradictory findings $[16,17]$, our study underlines that the contribution of the Hp2-2 type is significant but limited. It is only apparent when considering gender and is further found to be moderate in comparison to age and alcohol abuse (as evidenced by regression coefficients in Table 2).

Lack of replication between earlier investigations of the influence of the Hp2-2 type on phenotypic expression of the p.C282Y homozygous genotype may have several explanations. It notably might reflect differences in selection and size of the sample studied. Here, we enrolled a large number of expressive p.C282Y homozygous individuals; that is, individuals who displayed, at least, biological changes of the disease (increased transferrin saturation levels, with or without increased serum ferritin concentrations). This permitted powerful statistical analyses and careful evaluation of the relationship between the Hp2-2 genotype and the severity of iron overload. In this way, our results were similar to those initially reported by Vlierberghe et al. from a cohort of 167 p.C282Y homozygous patients [15]. In the overall population of patients, univariate analyses revealed that all the studied iron parameters were slightly 
TABLE 1

(a) Sociodemographic and biochemical characteristics of the 351 p.C282Y homozygous patients according to Hp genotype.

\begin{tabular}{lccc}
\hline Characteristic & \multicolumn{2}{c}{ Hp genotype } & Hp2-2 \\
& Hp1-1 & Hp2-1 & 126 \\
Sociodemographic & 46 & 179 & $64 \mathrm{M}, 62 \mathrm{~F}$ \\
$\quad$ Number of patients & $32 \mathrm{M}, 14 \mathrm{~F}$ & $99 \mathrm{M}, 80 \mathrm{~F}$ & $46.4 \pm 13.8$ \\
$\quad$ Gender (Males, Females) & $46.5 \pm 10.8$ & $47.4 \pm 13.8$ & 0.7887 \\
$\quad$ Age at diagnosis (years) & $76.8 \pm 15.1$ & $78.7 \pm 14.3$ & $80.0 \pm 12.9$ \\
Biochemical & $604[310-1136]$ & $570[280-1024]$ & $631[329-1196]$ \\
Transferrin saturation $(\%)$ & $3.6[1.8-6.1]$ & $3.3[1.8-5.6]$ & $3.9[2.0 .-6.8]$ \\
Serum ferritin $(\mu \mathrm{g} / \mathrm{L})$ & & 0.4009 \\
Iron removed $(\mathrm{g})^{1}$ & & 0.3580 \\
\hline
\end{tabular}

(b) Sociodemographic and biochemical characteristics of the studied 351 p.C282Y homozygous patients according to gender.

\begin{tabular}{lccc}
\hline Characteristic & \multicolumn{2}{c}{ Gender } \\
& Males & Females & \\
\hline Sociodemographic & & 156 \\
$\quad$ Number of patients & 195 & $47.5 \pm 14.5$ \\
$\quad$ Age at diagnosis (years) & $46.5 \pm 12.5$ & & 0.4854 \\
Biochemical & & $75.0 \pm 14.0$ \\
$\quad$ Transferrin saturation $(\%)$ & $82.0 \pm 13.1$ & $320[179-562]$ & $<0.0001$ \\
Serum ferritin $(\mu \mathrm{g} / \mathrm{L})$ & $892[553-1732]$ & $3.2[2.0-5.8]$ & $<0.0001$ \\
$\quad$ Iron removed $(\mathrm{g})^{1}$ & $4.9[3.4-8.2]$ & 0.0001 \\
\hline
\end{tabular}

(c) Sociodemographic and biochemical characteristics of the studied 351 p.C282Y homozygous patients according to alcohol consumption.

\begin{tabular}{|c|c|c|c|}
\hline \multirow{2}{*}{ Characteristic } & \multicolumn{3}{|c|}{ Alcohol consumption } \\
\hline & $\geq 60 \mathrm{~g} /$ day & $<60$ g/day & $P$ value \\
\hline \multicolumn{4}{|l|}{ Sociodemographic } \\
\hline Number of patients & 27 & 324 & \\
\hline Gender (Males, Females) & $25 \mathrm{M}, 2 \mathrm{~F}$ & $170 \mathrm{M}, 154 \mathrm{~F}$ & \\
\hline Age at diagnosis (years) & $48.9 \pm 12.5$ & $46.7 \pm 13.5$ & 0.4185 \\
\hline \multicolumn{4}{|l|}{ Biochemical } \\
\hline Transferrin saturation (\%) & $88.3 \pm 8.0$ & $78.1 \pm 14.0$ & 0.0002 \\
\hline Serum ferritin $(\mu \mathrm{g} / \mathrm{L})$ & $1256[658-2315]$ & 562 [283-967] & $<0.0001$ \\
\hline Iron removed $(\mathrm{g})^{1}$ & $6.8[4.8-9.7]$ & $3.4[1.8-5.9]$ & $<0.0001$ \\
\hline
\end{tabular}

${ }^{1} N=325$ (178 males and 147 females).

Age at diagnosis and transferrin saturation are expressed as mean \pm standard deviation.

Serum ferritin concentrations and iron removed by phlebotomy are presented as median and interquartile range.

increased in carriers of the Hp2-2 genotype. Stratification of the population by gender was, however, needed to obtain significant differences. These differences were restricted to the male subgroup, and to markers of body iron excess.

As HFE-related hemochromatosis develops slowly, gender and age are recognized as important modifying factors [3]. Menstruation and pregnancy, which deplete iron stores, act as temporary protective factors in women of reproductive age, whereas biological changes and subsequent clinical manifestations of the disease are commonly less marked in younger people than in older people. In addition, alcohol intake is known to worsen the hepatic damage produced by iron overload [21, 24]. We have previously shown that alcohol abuse (alcohol consumption $>60 \mathrm{~g}$ per day) results in higher iron parameters, and more frequent clinical manifestations [6]. To permit a more incisive examination of whether there is a true cause-and-effect relationship between the Hp2-2 genotype and increased iron parameters, we fitted multiple linear regression models with gender, but also age at diagnosis and alcohol abuse as potential confounders. These models led to the presentation of the Hp2-2 genotype as an independent factor increasing serum ferritin concentrations and iron removed by phlebotomy (Table 2). However, the models also showed that Hp2-2 was the lowest predictor. Adjusting for the strong effect of sex and age, carriers of the Hp2-2 genotype were thus less likely than alcohol abusers to have elevated serum ferritin and iron removed by phlebotomy. This latter point was verified in specific tests where medians for serum ferritin and iron removed by phlebotomy were significantly higher in alcohol abusers (who carried the Hp1-1 or Hp1-2 genotype) than in patients with the Hp2-2 genotype (who declared no excessive alcohol 
TABLE 2: Regression coefficients $(\beta)$ and 95\% confidence intervals (CIs) for variables influencing serum ferritin ( $\boldsymbol{\mu} \mathrm{g} / \mathrm{L}$; $\log _{10}$ transformed) and iron removed by phlebotomy (g; $\log _{10}$ transformed) in p.C282Y homozygous patients.

\begin{tabular}{|c|c|c|c|c|}
\hline \multirow[t]{2}{*}{ Variable } & \multicolumn{2}{|c|}{ Serum ferritin ${ }^{1}$} & \multicolumn{2}{|c|}{ Iron removed $^{2}$} \\
\hline & $10^{\beta}(95 \% \mathrm{CI})$ & $P$ value & $10^{\beta}(95 \% \mathrm{CI})$ & $P$ value \\
\hline Gender $^{3}$ & $0.33(0.27,0.39)$ & $<0.0001$ & $0.38(0.33,0.45)$ & $<0.0001$ \\
\hline \multicolumn{5}{|l|}{ Age at diagnosis ${ }^{4}$} \\
\hline Quartile 2 & $1.33(1.05,1.69)$ & 0.0204 & $1.27(1.02,1.58)$ & 0.0304 \\
\hline Quartile 3 & $1.77(1.39,2.26)$ & $<0.0001$ & $1.48(1.19,1.84)$ & 0.0005 \\
\hline Quartile 4 & $1.88(1.47,2.39)$ & $<0.0001$ & $1.63(1.30,2.03)$ & $<0.0001$ \\
\hline Alcohol consumption ${ }^{5}$ & $1.57(1.13,2.18)$ & 0.0073 & $1.53(1.11,2.12)$ & 0.0096 \\
\hline Hp group ${ }^{6}$ & $1.31(1.09,1.57)$ & 0.0033 & $1.29(1.09,1.52)$ & 0.0024 \\
\hline
\end{tabular}

${ }^{1} N=351$ (195 males and 156 females). Adjusted $r^{2}=38.3 \%$.

${ }^{2} N=325$ (178 males and 147 females). Adjusted $r^{2}=36.1 \%$.

${ }^{3}$ Male sex was used as the reference category.

${ }^{4}$ Quartile 1 was used as the reference category.

${ }^{5}$ Daily alcohol intake $<60 \mathrm{~g}$ was used as the reference category.

${ }^{6} \mathrm{Hp} 1-1$ and $\mathrm{Hp} 2-1$ genotypes were grouped and used as the reference category.

TABLe 3

(a) Regression coefficients $(\beta)$ and $95 \%$ confidence intervals (CIs) for variables influencing serum ferritin ( $\mu \mathrm{g} / \mathrm{L} ; \log _{10}$-transformed) and iron removed by phlebotomy ( $\mathrm{g}$; $\log _{10}$-transformed) in p.C282Y homozygous males patients.

\begin{tabular}{|c|c|c|c|c|}
\hline \multirow[t]{2}{*}{ Variable } & \multicolumn{2}{|c|}{ Serum ferritin $^{1}$} & \multicolumn{2}{|c|}{ Iron removed ${ }^{2}$} \\
\hline & $10^{\beta}(95 \% \mathrm{CI})$ & $P$ value & $10^{\beta}(95 \% \mathrm{CI})$ & $P$ value \\
\hline \multicolumn{5}{|l|}{ Age at diagnosis ${ }^{3}$} \\
\hline Quartile 2 & $1.30(0.99,1.71)$ & 0.0603 & $1.29(0.99,1.66)$ & 0.0567 \\
\hline Quartile 3 & $1.87(1.39,2.51)$ & $<0.0001$ & $1.39(1.05,1.68)$ & 0.0204 \\
\hline Quartile 4 & $1.59(1.19,2.13)$ & 0.0019 & $1.50(1.15,1.96)$ & 0.0032 \\
\hline Alcohol consumption ${ }^{4}$ & $1.59(1.18,2.16)$ & 0.0027 & $1.50(1.11,2.04)$ & 0.0096 \\
\hline Hp group 5 & $1.40(1.13,1.74)$ & 0.0026 & $1.38(1.12,1.69)$ & 0.0022 \\
\hline
\end{tabular}

(b) Regression coefficients $(\beta)$ and $95 \%$ confidence intervals (CIs) for variables influencing serum ferritin $\left(\mu \mathrm{g} / \mathrm{L}\right.$; $\log _{10}$-transformed) and iron removed by phlebotomy ( $\mathrm{g}$; $\log _{10}$-transformed) in p.C282Y homozygous females patients.

\begin{tabular}{|c|c|c|c|c|}
\hline \multirow[t]{2}{*}{ Variable } & \multicolumn{2}{|c|}{ Serum ferritin $^{1}$} & \multicolumn{2}{|c|}{ Iron removed $^{2}$} \\
\hline & $10^{\beta}(95 \% \mathrm{CI})$ & $P$ value & $10^{\beta}(95 \% \mathrm{CI})$ & $P$ value \\
\hline \multicolumn{5}{|l|}{ Age at diagnosis ${ }^{3}$} \\
\hline Quartile 2 & $1.26(0.83,1.89)$ & 0.2723 & $1.31(0.90,1.91)$ & 0.1574 \\
\hline Quartile 3 & $1.85(1.23,2.78)$ & 0.0033 & $1.58(1.09,2.29)$ & 0.0154 \\
\hline Quartile 4 & $2.38(1.58,3.59)$ & $<0.0001$ & $1.87(1.29,2.71)$ & 0.0012 \\
\hline Alcohol consumption ${ }^{4}$ & $1.37(0.37,5.01)$ & 0.6351 & $1.65(0.53,5.15)$ & 0.3892 \\
\hline Hp group ${ }^{5}$ & $1.20(0.89,1.61)$ & 0.2383 & $1.17(0.90,1.53)$ & 0.2457 \\
\hline
\end{tabular}

${ }^{1} \mathrm{~N}=195$. Adjusted $\mathrm{r}^{2}=16.2 \%$.

${ }^{2} \mathrm{~N}=178$. Adjusted $\mathrm{r}^{2}=13.5 \%$.

${ }^{3}$ Quartile 1 was used as the reference category.

${ }^{4}$ Daily alcohol intake $<60 \mathrm{~g}$ was used as the reference category.

${ }^{5} \mathrm{Hp} 1-1$ and $\mathrm{Hp} 2-1$ genotypes were grouped and used as the reference category.

intake). The observed values were $1256.0 \mu \mathrm{g} / \mathrm{L}[\mathrm{Q} 1=803.0$, $\mathrm{Q} 3=2311.0]$ versus $599.0 \mu \mathrm{g} / \mathrm{L}[\mathrm{Q} 1=319.5 ; \mathrm{Q} 3=975.0]$ $(P=.0010)$ and $6.6 \mathrm{~g}[\mathrm{Q} 1=4.8 ; \mathrm{Q} 3=9.7]$ versus $3.6 \mathrm{~g}[\mathrm{Q} 1=$ 1.8 ; $\mathrm{Q} 3=6.8](P=.0055)$, repectively.

We conclude that, by accentuating the iron burden, the frequent Hp2-2 genotype could explain some of the phenotypic heterogeneity seen in p.C282Y homozygous hemochromatosis patients. However, we support the idea that this influence is weak, and that other genetic and environmental modifying factors should be considered, to efficiently distinguish those homozygous patients at higher risk of developing morbid complications.

\section{Acknowledgments}

This work was supported by grants from the Centre Hospitalier Universitaire de Brest (Programme Hospitalier 
de Recherche Clinique), by the conseil scientifique de l'Etablissement Français du Sang (projet 2003.19), and by the Fondation pour la Recherche Médicale.

\section{References}

[1] P. C. Adams, P. Brissot, and L. W. Powell, "EASL International consensus conference on haemochromatosis," Journal of Hepatology, vol. 33, no. 3, pp. 485-504, 2000.

[2] A. Bomford, "Genetics of haemochromatosis," The Lancet, vol. 360, no. 9346, pp. 1673-1681, 2002.

[3] A. Pietrangelo, "Hereditary hemochromatosis—a new look at an old disease," The New England Journal of Medicine, vol. 350, no. 23, pp. 2383-2397, 2004.

[4] G. Le Gac and C. Férec, "The molecular genetics of haemochromatosis," European Journal of Human Genetics, vol. 13, no. 11, pp. 1172-1185, 2005.

[5] T. H. Bothwell, R. W. Charlton, and A. G. Motulsky, "Hemochromatosis," in The Metabolic and Molecular Bases of Inherited Diseases, C. R. Scriver, A. L. Beaudet, W. S. Sly, and D. Valle, Eds., pp. 2237-2269, McGraw-Hill, New York, NY, USA, 1995.

[6] V. Scotet, M.-C. Mérour, A.-Y. Mercier, et al., "Hereditary hemochromatosis: effect of excessive alcohol consumption on disease expression in patients homozygous for the C282Y mutation," American Journal of Epidemiology, vol. 158, no. 2, pp. 129-134, 2003.

[7] J. Waalen, B. G. Nordestgaard, and E. Beutler, "The penetrance of hereditary hemochromatosis," Best Practice \& Research Clinical Haematology, vol. 18, no. 2, pp. 203-220, 2005.

[8] L. W. Powell, V. N. Subramaniam, and T. R. Yapp, "Haemochromatosis in the new millennium," Journal of Hepatology, vol. 32, pp. 48-62, 2000.

[9] A. Lazarescu, B. M. Snively, and P. C. Adams, "Phenotype variation in $\mathrm{C} 282 \mathrm{Y}$ homozygotes for the hemochromatosis gene," Clinical Gastroenterology and Hepatology, vol. 3, no. 10, pp. 1043-1046, 2005.

[10] P. Ascenzi, A. Bocedi, P. Visca, et al., "Hemoglobin and heme scavenging," IUBMB Life, vol. 57, no. 11, pp. 749-759, 2005.

[11] J. R. Delanghe and M. R. Langlois, "Haptoglobin polymorphism and body iron stores," Clinical Chemistry and Laboratory Medicine, vol. 40, no. 3, pp. 212-216, 2002.

[12] K. B. Wicher and E. Fries, "Prohaptoglobin is proteolytically in the endoplasmic reticulum by the complement C1r-like protein," Proceedings of the National Academy of Sciences of the United States of America, vol. 101, no. 40, pp. 14390-14395, 2004.

[13] M. Kristiansen, J. H. Graversen, C. Jacobsen, et al., "Identification of the haemoglobin scavenger receptor," Nature, vol. 409, pp. 198-201, 2001.

[14] M. R. Langlois, M.-E. Martin, J. R. Boelaert, et al., "The haptoglobin 2-2 phenotype affects serum markers of iron status in healthy males," Clinical Chemistry, vol. 46, no. 10, pp. 1619-1625, 2000.

[15] H. Van Vlierberghe, M. Langlois, J. Delanghe, et al., "Haptoglobin phenotype 2-2 overrepresentation in Cys282Tyr hemochromatotic patients," Journal of Hepatology, vol. 35, no. 6, pp. 707-711, 2001.

[16] E. Beutler, T. Gelbart, and P. Lee, "Haptoglobin polymorphism and iron homeostasis," Clinical Chemistry, vol. 48, no. 12, pp. 2232-2235, 2002.

[17] K. Carter, D. J. Bowen, C. A. McCune, and M. Worwood, "Haptoglobin type neither influences iron accumulation in normal subjects nor predicts clinical presentation in HFE C282Y haemochromatosis: phenotype and genotype analysis," British Journal of Haematology, vol. 122, no. 2, pp. 326-332, 2003.

[18] E. Tolosano, S. Fagoonee, C. Garuti, et al., "Haptoglobin modifies the hemochromatosis phenotype in mice," Blood, vol. 105, no. 8, pp. 3353-3355, 2005.

[19] S. Jacolot, G. Le Gac, V. Scotet, I. Quere, C. Mura, and C. Ferec, "HAMP as a modifier gene that increases the phenotypic expression of the HFE pC282Y homozygous genotype," Blood, vol. 103, no. 7, pp. 2835-2840, 2004.

[20] G. Le Gac, V. Scotet, C. Ka, et al., "The recently identified type 2A juvenile haemochromatosis gene $(H J V)$, a second candidate modifier of the C282Y homozygous phenotype," Human Molecular Genetics, vol. 13, no. 17, pp. 1913-1918, 2004.

[21] L. M. Fletcher, J. L. Dixon, D. M. Purdie, et al., "Excess alcohol greatly increases the prevalence of cirrhosis in hereditary hemochromatosis," Gastroenterology, vol. 122, no. 2, pp. 281289, 2002.

[22] W. Koch, W. Latz, M. Eichinger, et al., "Genotyping of the common haptoglobin Hp 1/2 polymorphism based on PCR," Clinical Chemistry, vol. 48, no. 9, pp. 1377-1382, 2002.

[23] M. R. Langlois and J. R. Delanghe, "Biological and clinical significance of haptoglobin polymorphism in humans," Clinical Chemistry, vol. 42, no. 10, pp. 1589-1600, 1996.

[24] P. C. Adams and S. Agnew, "Alcoholism in hereditary hemochromatosis revisited: prevalence and clinical consequences among homozygous siblings," Hepatology, vol. 23, no. 4, pp. 724-727, 1996. 


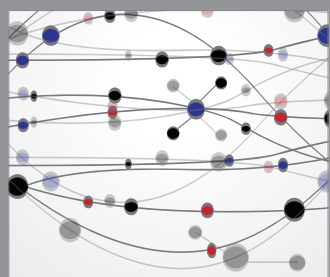

The Scientific World Journal
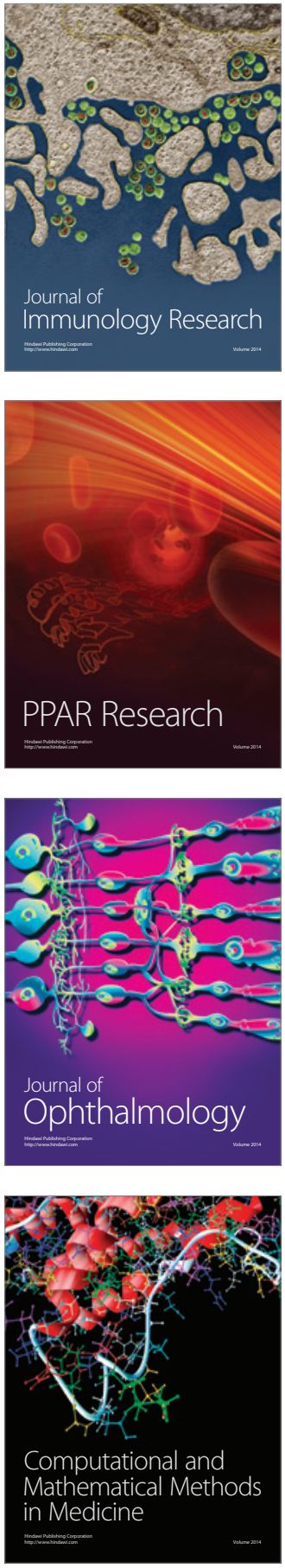

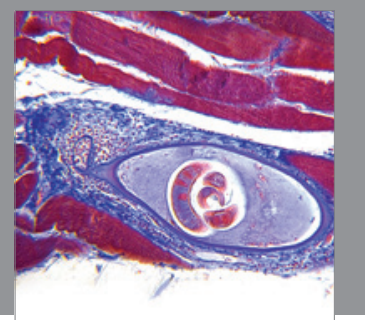

Gastroenterology

Research and Practice
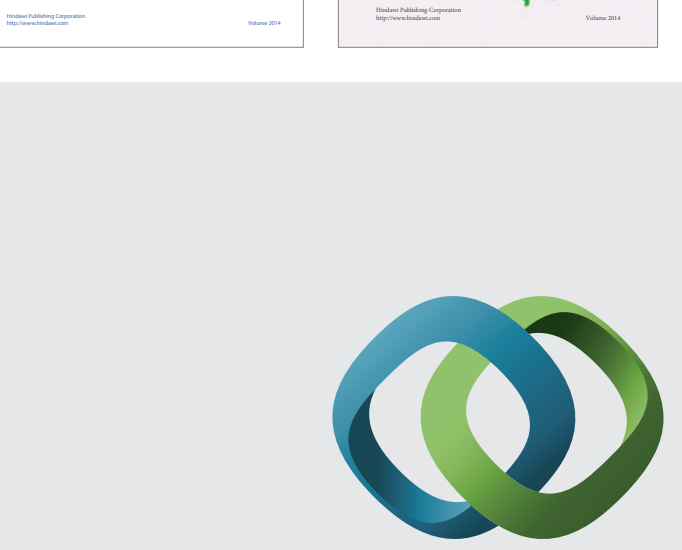

\section{Hindawi}

Submit your manuscripts at

http://www.hindawi.com
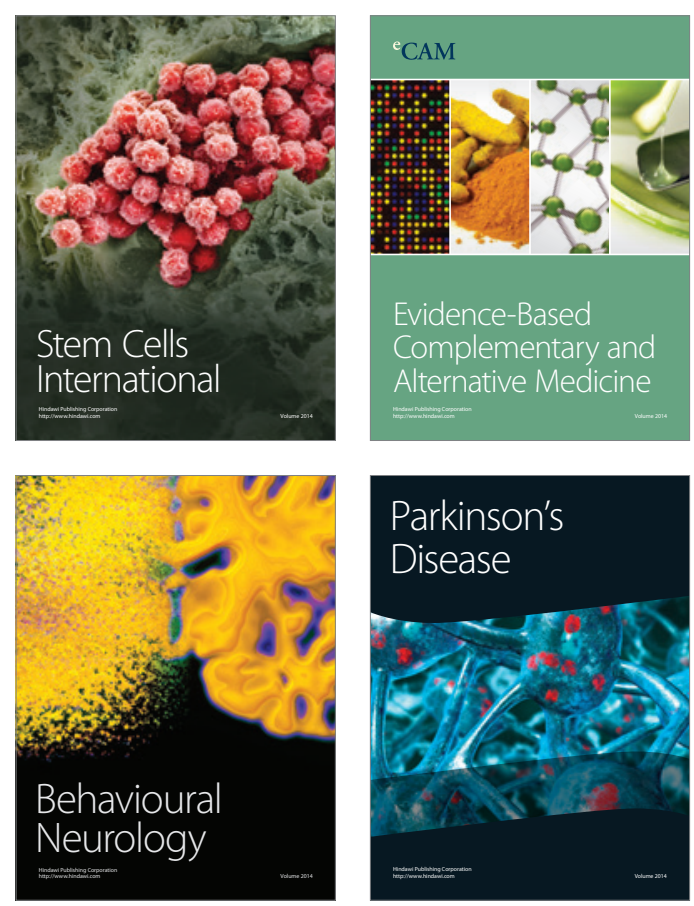

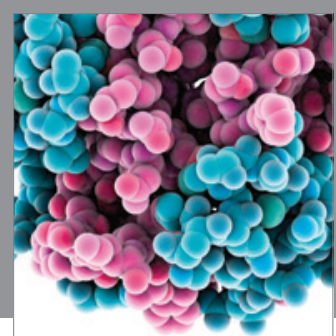

Journal of
Diabetes Research

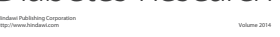

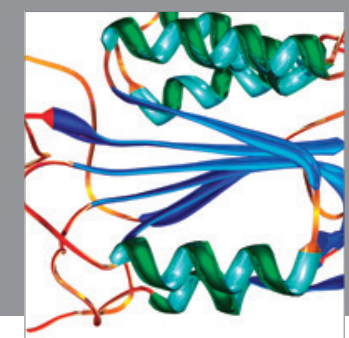

Disease Markers
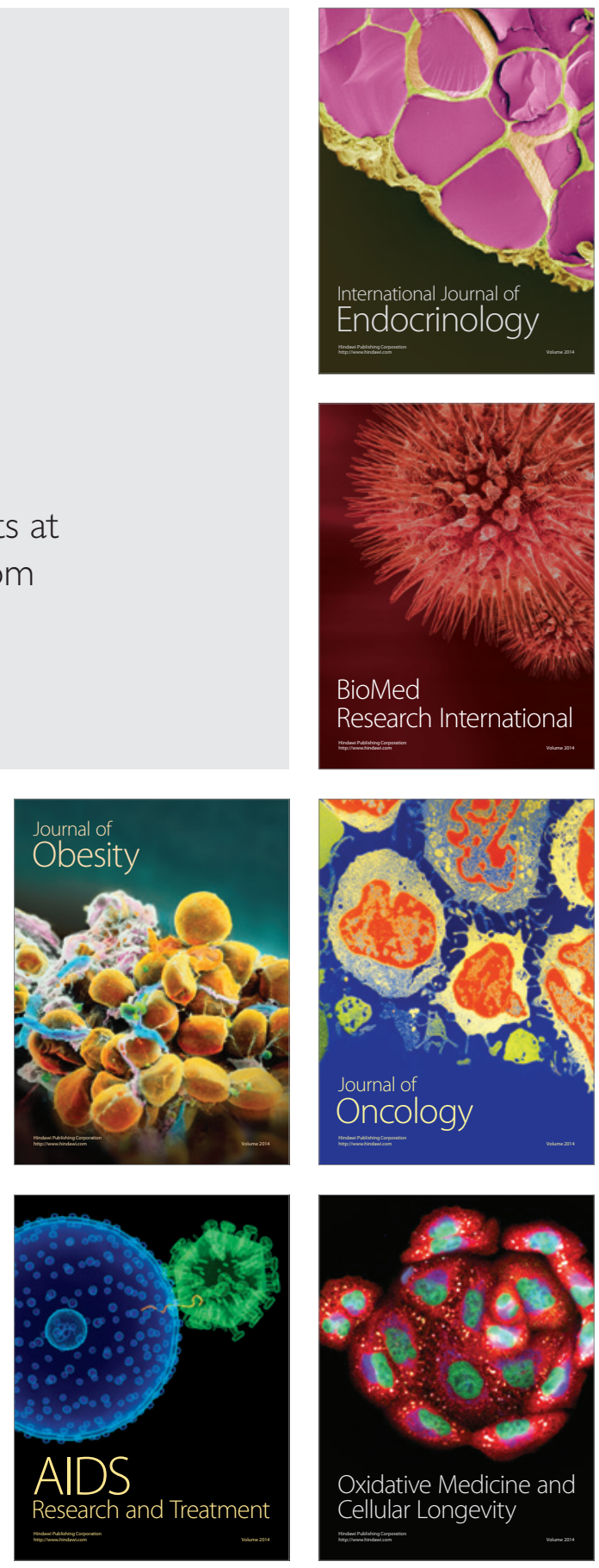